\title{
An Evolving Network Model of Self-employment Agglomeration
}

\author{
Yong Tang ${ }^{1}$ \\ ${ }^{1}$ Urban Management Department, Hunan City University, Yiyang, P. R. China \\ Correspondence: Yong Tang, Urban Management Department, Hunan City University, Yiyang, P. R. China. Tel: \\ 86-731-635-3164.E-mail: tyzyhtt@126.com
}

Received: July 15, 2015

Accepted: July 29, 2015

Online Published: August 4, 2015

doi:10.5430/bmr.v4n3p15

URL: http://dx.doi.org/10.5430/bmr.v4n3p15

\begin{abstract}
Self-employment agglomeration is a very typical entrepreneurship in China's urbanization process, and new comers can easily obtain resource aids from agglomeration because the communications there are very frequent. Referring to complex network theory, we construct an evolving network which can basically reflect the situation of agglomeration evolution in China. Under given preferential attachment rule, we simulate its evolving process and calculate its statistic properties. We find that self-employment agglomeration with this evolving rule has comparatively big average degree and power-law degree distribution; it has large clustering coefficient and short average path length. We conclude that self-employment agglomeration has the properties both in scale-free network and in small world network. The mechanisms of statistic properties on self-employment are also analyzed.
\end{abstract}

Keywords: Self-employment Agglomeration, Preferential Attachment, Complex Network, Complete Graph

\section{Introduction}

In recent years, with the deepening of China's new-type urbanization policies, millions of farmers and city registered lay-offs choose entrepreneurship, mainly as necessity entrepreneurs (Hernandez, 2012), to substitute the fierce competition in employment markets. Most of these kinds of entrepreneurship are characterized as small scale, most 2-5 members; and low investment, often less than 500000 RMB (Yong T., 2011). As for the inner governance, most of these kinds of entrepreneurial entities are regulated by members together; hence they have no strict hierarchy and detailed responsibilities in controlling. Under this kind of flexible entity, every member is free to enter or leave his post in order to running for more lucrative businesses. So, this kind of entity is easily connected with other entities along with the repeated entrance of members. From network perspective, every entity is a small network, often fully connected; and lots of entities are interconnected to form a bigger network, often shown as agglomeration. This kind of agglomeration based on self-employment is widely seen in China's town level, where most of businesses are operated by farmers and lay-off workers, and all businesses are agglomerated in an area of less than $1 \mathrm{~km} 2$, and almost all entrepreneurs are known to each other, so they are prone to seek resources support from adjacent entities.

Seeing this phenomenon, some policy makers welcome its flourish because it may create "popular entrepreneurship and innovation" (twin engines encouraged by Chinese authorities to sustain China's sustainable development). However, some scholars are questioning its function on economic development because this agglomeration is hidden by some self employment entrepreneurship followers, who are only imitating prior entrepreneurs' behaviors. Confined by imitation, most of nascent entrepreneurs lack innovative ability and good market consciousness. Under this situation, many entrepreneurs are locked in this agglomeration, hardly resisting randomly emerged market risks. Thus, big scale agglomeration will lead to inefficient investment and low level innovation.

In order to solve the paradox above, we think it is necessary to explore the mechanism of network formation and function, so as to recognize the dynamics of self employment agglomeration. From dynamic perspective, we think the key is to explore the rational of network evolution. In this paper, we will conduct our research using complex theory, and focus on solving following questions: (1) how can this self employment agglomeration be formed? (2) what network properties does this kind of agglomeration have? And (3) how does these properties affect self-employment activities. 


\section{Literature Review}

Entrepreneurial agglomeration is a very important phenomenon in the economic world. Silicon Valley in U.S. and Yiwu in China are very typical entrepreneurial agglomerations, both of which contain thousands of industries and entrepreneurs and are shouldering the responsibilities of creating regional entrepreneurial atmosphere and diffusing entrepreneurial knowledge to nascent companies (Rosenthal \& Strange, 2003). However, academic circle and administrative authorities are facing the dilemma that entrepreneurship flourishes in some regions and not in others, though most of the conditions are similar to each other in the preliminary phase. Some researches provided theoretical analysis from social advantages and economic advantages (Puga \& Venables, 1996; Kim et al., 2006; Savvas et al., 2009).

Researchers found that entrepreneurial activity does not occur in vacuum, but instead is rooted in cultural and social contexts, specifically within personal and institutional networks (Kloosterman, 2010). For diversified resource requirement in entrepreneurship, there are growing researches suggesting the importance of networks to facilitate the entrepreneurs to get resources, which makes network another significant source of advantage for entrepreneurs (Audretsch \& Keilbach, 2004; Davidson \& Honig, 2003). Studies indicate that networking allows entrepreneurs to enlarge their knowledge of opportunities, to gain access to critical resources, and to resist business obstacles (Wiklund et al., 2011).

However, network may also bring some disadvantages to entrepreneurs. When a new comer enters into a new network, he may pay lots of cost to seek for appropriate resources needed, and even more cost on linking these necessary resources (Hoang \& Yi, 2015). Self-employer is less capable in seeking for and bonding new resources within key network actors because of its small investment and weak social capital. Then, network may misguide innovative clues (Yang et al., 2012). A formed network can contain stable and specific rules, belief and even culture, which could produce path-dependence for new comers (Dobusch \& Schüßler, 2013). And last, network has some statistic properties, some of which can determine the robustness or fragileness of resource communication (Ferriani et al., 2009). Though some scholars find that an entrepreneurial network has some properties similar to complex network, it is unclear that self employment agglomeration may be classified as another kind of complex network.

But self employment agglomeration is also possible. Under the non-regulated organization, self-employment entrepreneurs are not constrained by strict regulations, but by the resources that members can provide. So, every new member who owns resources necessary for entrepreneurship is welcomed, and the new member will not feel inferior because he is immediately becoming an unseparated part for the wholeness of entrepreneurship (Tang, 2013). From easiness of communication, self employers in similar circle are more easily fully connectedly than other entrepreneurs (Katz \& Williams, 1997), which probably produce fully connected network for some given self employers .

Form prior literatures we find that some scholars have conducted research from entrepreneurial agglomeration, entrepreneurial network, and self employment agglomeration. Some researches have found that entrepreneurial network may be analyzed from its structure statistics because it can be seen as specific complex network. However, prior researches seldom discuss self employment from complex network statistics, and ignore the network dynamics in acquiring resources in a self employment agglomeration. For the reason that self employment is easily fully connected, we can construct an evolving network when we set the entrance rules for every new self employer.

\section{Self-employment Agglomeration Network Evolving Rule}

We firstly analyze the evolving process of a self employment agglomeration. Considering the evolution of an entrepreneurship, a new entrant's decision on entering this entrepreneurship will make this network grow and further fully interconnected. It is worth noting that a new entrant may enter into more than 1 network simultaneously (such as a new entrant may firstly enter into a network of capital provider, then enter into a network of technology provider; from network perspective, these two small networks or subnetworks are connected by the new entrant), thus he who is in one network may builds ties with other members in other networks. From wholeness, we can construct an agglomeration network comprised by lots of smaller networks. Fig. 1 is the topologies of 2 self-employment agglomerations in China, in which most of actors have more than 3 connections with other members and they have connections from inner small network to intra networks. From dynamic perspective, we can assume these kinds of agglomerations are in growth, i.e., every member can be seen as a new one with preferential attachment to existing small network, and repeated entrance will cluster lots of new comers, so the agglomeration is formed. 


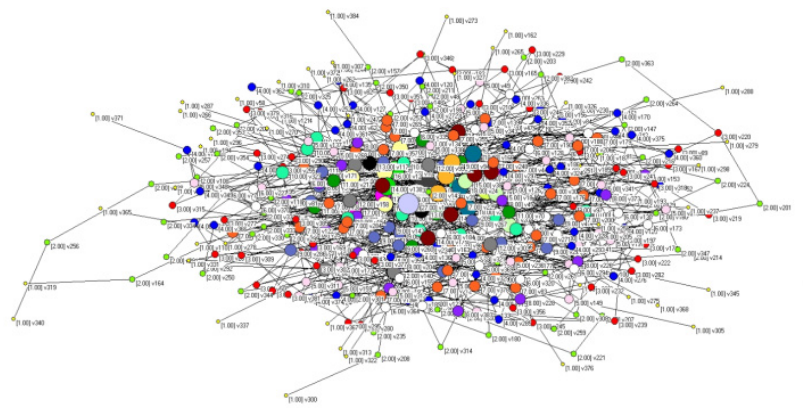

(a)

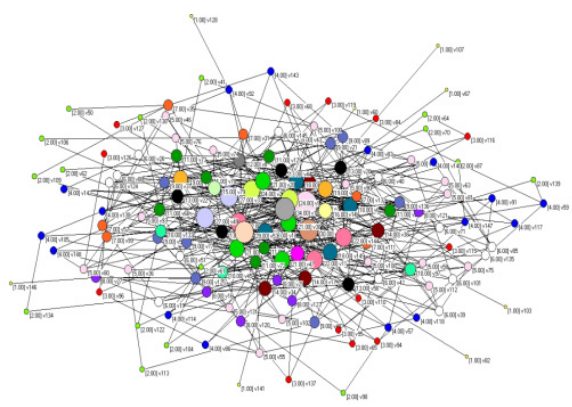

(b)

Figure 1. Topologies of two self-employment agglomerations in China

Note: (a) is a self-employment agglomeration in a town in Changsha City, Hunan Province, China, whose members are 385; (b) is a self-employment agglomeration in a town in Yiyang City, Hunan Province, China, whose members are 149.

For simplicity, we suppose an agglomeration is on its evolving process, and it has the preferential attachment of randomly selected $S$-vertex complete graph in it. Then this agglomeration is an evolving network, and the evolving rules are as below:

Step 1: suppose in a self-employment small network, $S$ members $(s \leq 5)$ are fully connected; here, $S$ members can be seen as $S$ vertices in network terminology. See fig. 1(a);

Step 2: a new member joins this small network, and he randomly chooses a $S$-vertex fully connected subnetwork to build ties with, so this member will add one vertex and $s$ edges to the network. See fig. 1(b);

Step 3: new members will repeat the preferential attachments to a $S$-vertex fully connected subnetwork in the whole network, and every new member will add 1 vertex and $S$ edges to the previous whole network. With this repetition, an agglomeration of self-employment emerges. The evolution process of time step 0-5 can be presented in fig 2(a)-(f).

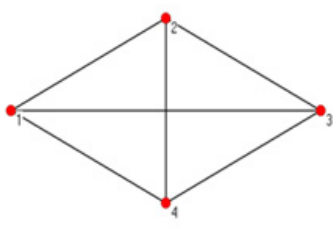

(a) $\mathrm{t}=0$

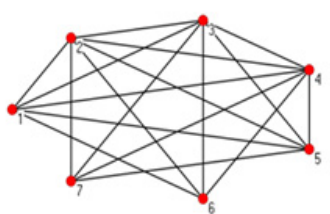

(d) $t=3$

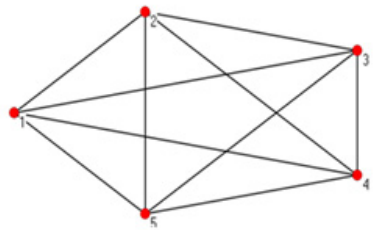

(b) $\mathrm{t}=1$

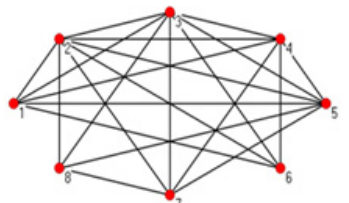

(e) $\mathrm{t}=4$

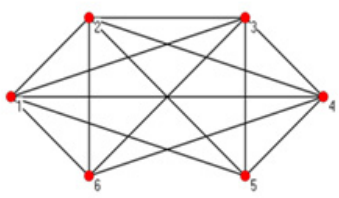

(c) $t=2$

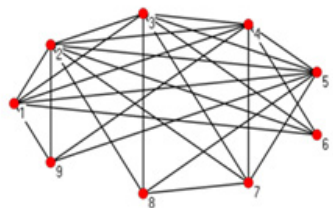

(f) $t=5$

Figure 2. The evolution of self-employment agglomeration $(s=4)$

We use simulation to draw the topology of an evolving network with 200 vertices under the 4-vertex complete graph attachment rule. As in fig. 3, we know that every vertex has at least 4 edges with other vertices, and the whole network has comparatively high density. For the vertices in the center, they have very dense connections with other vertices, indicating that they have frequent resource communication within their own entities and with other entities. In network theory, they are often seen as overlapping vertices because they will appear in various entities. 


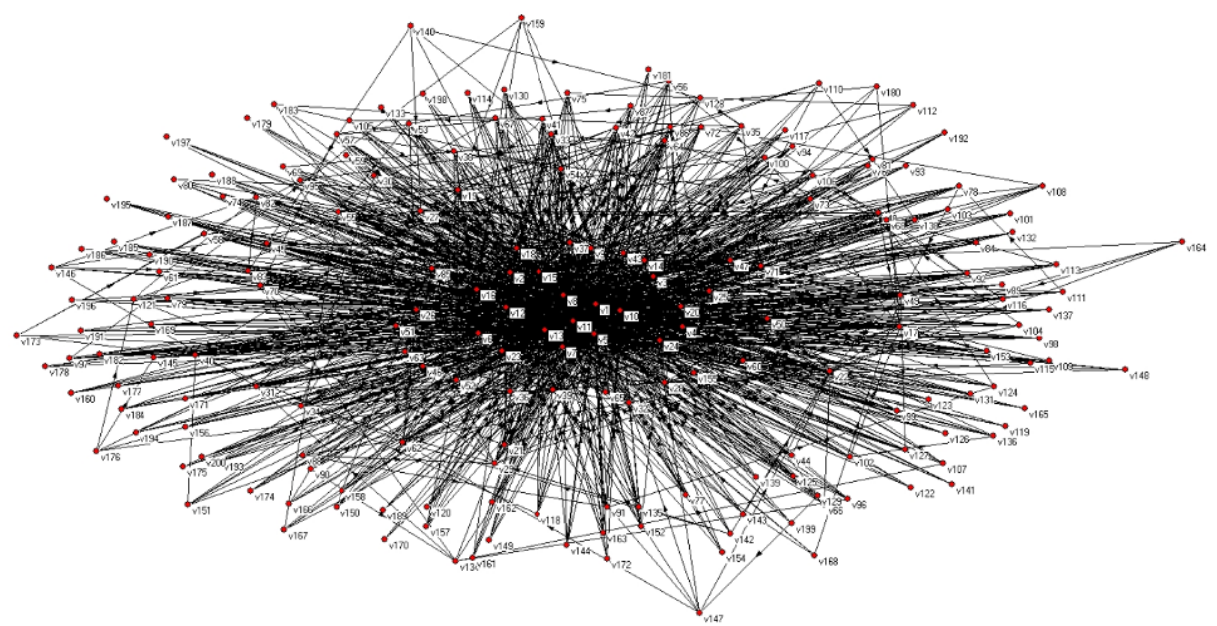

Figure 3. The topology of evolving network ( $s=4$, vertices $=200$ )

\section{Agglomeration Network Statistic Properties}

Though we can simulate the topology of self-employment agglomeration, we cannot tell the dynamics of its evolution under given rules. This section we will discuss some statistic properties from network dynamic perspective.

Considering most of the self-employment entities are in small scale, we suppose every entity is with the same scale of $s$ members. We define $\mathrm{G}$ as a $s^{-}$complete graph. Under the evolving rule set in section 2 , we know that in time 0 , there exists a fully connected network $G=(V, E)$, and $|V|=s,|E|=\frac{s(s-1)}{2}$. A new vertex will attach a randomly selected $s^{-}$complete graph. We then calculate average degree, degree distribution, clustering coefficient and average path length of this evolving network (Albert R, Barabási A L., 2002).

\subsection{Average degree}

Average degree tells us the wholeness of resource communication in an agglomeration. Suppose an $s^{-}$complete graph in time step 0 ; then we add one vertex to this graph, so number of edges will be $\frac{s(s-1)}{2}+s$ and number of total degree will be $2\left(\frac{s(s-1)}{2}+s\right)$. In time step $v$, there will be $v+s$ vertices, and $\frac{s(s-1)}{2}+v s$ edges. Total degree will be $2\left(\frac{s(s-1)}{2}+v s\right)$, so average degree is $\frac{2\left(\frac{s(s-1)}{2}+v s\right)}{v}$. If $v$ is big enough, the average degree is approaching to $2 s$. Hence, we can see self-employment agglomeration with this evolving rule has comparatively big average degree, which is beneficial for resource sharing in entrepreneurship. It's worth noting that though the quantity of resource communication will not be improved with the growth of agglomeration, the quality will be enhanced significantly because new members will bring more heterogeneous resources that essential for sustainable entrepreneurship (Alvarez S A, Busenitz L W., 2001).

4.2 Degree distribution

Degree distribution is a very important statistic property for network research. For a network of entrepreneurial agglomeration, degree distribution can be used to categorize network type, and predict the entrepreneurial performance based on this kind of network.

It is easily assumed that the vertex with higher degree will appear in $s^{-}$complete graph with higher probability. So we can calculate the evolving mode of degree $k_{v}$ for any given vertex $v$ using mean field theory (Barabási A L. et al., 1999):

$$
\frac{\partial k_{v}}{\partial t}=\frac{(s-1) k_{v}-s(s-2)}{s t+1}
$$

Where, denominator is the number of complete graph in the initial state, and numerator is the number of complete 
graphs centered on vertex $v$. In time $t_{v}$, this vertex is attached to whole network, when $k_{v}\left(t_{v}\right)=s$ is the initial condition. We refer to Zhang Z Z., et al. (2005) to get the solution for (1):

$$
k_{v}(t)=s(s-2) \frac{1}{s-1}+\frac{s}{s-1}\left(\frac{s t+1}{s t_{v}+1}\right)^{1-\frac{1}{s}}
$$

If we set $\beta=1-\frac{1}{S}$, (2) expresses the degrees of any vertices will evolve following the same path. Hence, accumulation degree probability function can be expressed as:

$$
P\left(k_{v}(t)<k\right)=P\left(t_{v}>\frac{s t+1}{s\left(1-\frac{1}{s}\right)^{1 / \beta}\left(k-\frac{s(s-2)}{s-1}\right)^{1 / \beta}}-\frac{1}{s}\right)
$$

As for vertex probability distribution, we introduce a vertex in very time step. Then, the probability function is:

$$
P_{v}\left(t_{v}\right)=1 /(s+t)
$$

Substituting (4) into function (3), we obtain:

$$
P\left(k_{v}(t)<k\right)=1-\mathrm{P}\left(t_{v} \leq \frac{s t+1}{s\left(1-\frac{1}{s}\right)^{1 / \beta}\left(k-s \frac{s-2}{s-1}\right)^{1 / \beta}}-\frac{1}{s}\right)
$$

Its equivalent function is:

$$
P\left(k_{v}(t)<k\right)=1-P\left(t_{v} \leq \frac{s t+1}{(s+t) s\left(1-\frac{1}{s}\right)^{1 / \beta}\left(k-\frac{s(s-2)}{s-1}\right)^{1 / \beta}}-\frac{1}{(s+t) s}\right)
$$

Notice that:

$$
P(k)=\frac{\partial P\left(k_{v}(t)<k\right.}{\partial k}
$$

We then have:

$$
P(k)=\frac{(s t+1)}{(s+t) s}\left(\frac{s}{s-1}\right)^{\frac{2 s-1}{s-1}}\left(k-\frac{s(s-2)}{s-1}\right)^{-\left(\frac{1}{\beta}+1\right)}
$$

We now discuss the power-law distribution of $P(k)$. Set $\gamma=\frac{1}{\beta}+1=\frac{2 s-1}{s-1}$, (8) can be rewritten as (9):

$$
P(k) \square\left(\frac{s}{s-1}\right)^{\gamma}\left(k-\frac{s(s-2)}{s-1}\right)^{-\gamma}
$$

Obviously, we have:

$$
\beta(\gamma-1)=1
$$

Where the relation $\beta(\gamma-1)=1$ between exponents is universal in scale-free network (Dorogovtsev S N., 2000).

We then simulate the degree distribution.

We set $\mathrm{s}=3$ and $\mathrm{s}=4$, and run a network with 100000 vertices. From (9) we know that $\gamma$ is 2.5 and 2.33. The simulation results are presented in fig. 4. 


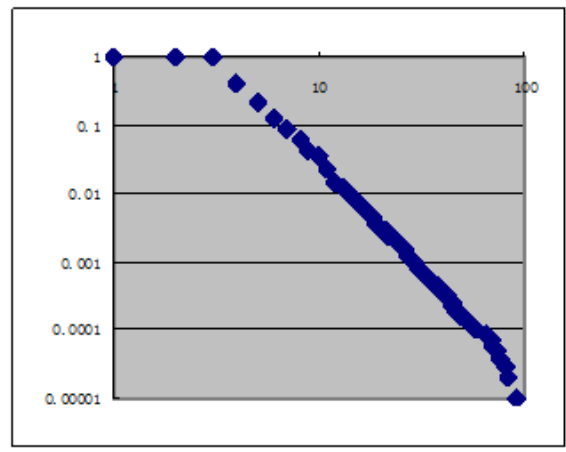

(a)

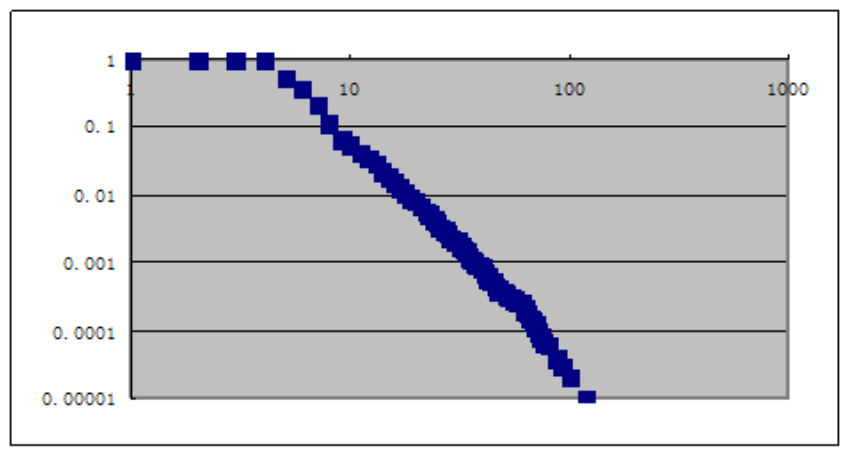

(b)

Figure 4. The degree distribution

Note: according to attachment rules, every vertex has at least $s$ degree, so in fig. $1, P(1)=P(2)=P(3)=1$; and in fig. 2, $P(1)=P(2)=P(3)=P(4)=1$. When we consider the power-law distribution, we need to concentrate on the interval of $[s,+\infty)$.

From the analysis we know that an agglomeration with given attachment rules has the power-law degree distribution, and the simulation of degree distribution appropriately reflects this property (both (a) and (b) are approximately linear distributed, and their slopes are approaching to -2.5 and -2.33), so it belongs to scale-free network. According to Aldrich \& Kim (2007), this kind of network is efficient in promoting resource communication and improving entrepreneurial performance.

\subsection{Clustering coefficient}

According to complex network statistics, clustering coefficient is defined as $C_{v}=\frac{\text { number of triangles connected to vertex }}{\text { number of triples centered on vertex }}$. Then the clustering coefficient for the whole network is the average: $C=\frac{1}{n} \sum_{v} C_{v}$. Now we move to the clustering coefficient of vertex $v$ which has the degree of $k$. When a new vertex attaches to a complete graph $s^{-}$, its degree is $m$, and $C_{v}=1$. In the next time steps, when new vertex considers to attach vertex $n$ in the whole network, it must attach to $s-1$ vertices that vertex $n$ connects with. Therefore, we can find that the degree of vertex is correlated to its clustering coefficient, and the relation can be expressed as:

$$
C(k)=\frac{\frac{s(s-1)}{2}+(s-1)(k-s)}{\frac{k(k-1)}{2}}=\frac{(s-1)(2 k-s)}{k(k-1)}
$$

Suppose degree $k$ is continuous, then the clustering coefficient of whole network is denoted as:

$$
C=\sum_{k} C(k) P(k)=\sum_{k=s+1}^{\infty} \frac{(s-1)(2 k-s)}{k(k-1)}\left(\frac{s}{s-1}\right)^{\gamma}\left(k-\frac{s(s-2)}{s-1}\right)^{-\gamma}
$$

Considering $C^{\prime}(s)>0, C(s)$ is an increasing function. When we set $s=4, C=0.811$, thus $\forall s>4, C(s)>0.811$. Hence, whole network with preferential attachment rules in this paper has very large clustering coefficient.

Large clustering coefficient is a very typical property of small world network. Generally speaking, self-employment agglomeration is beneficial for resource sharing, which is essential for the entrepreneurs because most of entrepreneurs there are facing the resource insufficiency. From another hand, intensive communication among entities is not appropriate for digging out entrepreneurial opportunities because of network embeddedness (Jack \& Anderson, 2002). In self-employment agglomeration, lots of members will have no impetus to seek out new ideas or innovate in their entrepreneurial behaviors. Thus, self-employment agglomeration may contain lots of necessity entrepreneurial behaviors, which are characterized on coping and repeating prior businesses, less able in fully exploring and exploiting the business efficiency and effectiveness.

\subsection{Average path length}

Path length denotes the easiness of one member connecting to others members in agglomeration, shorter path length implying one can easily get aids from others. As for average path length, considering an undirected network, and let us define $d(i, j)$ is the shortest distance between vertex pair $(i, j)$, also known as geodesic distance of $i-j$. Then 
the total distance in a $N$ vertex whole network can be denoted as:

$$
\sigma(M)=\sum_{0 \leq i \leq j \leq M-1} d(i, j)
$$

Hence, the average path length is:

$$
L(M)=\frac{2 \sigma(M)}{M(M-1)}
$$

Given a network, we find that a new vertex will add distance to the network, but it will not affect the total distance of existing network. The relation of distance of an $M$ vertex whole network and an $M+1$ vertex whole network is denoted by:

$$
\sigma(M+1)=\sigma(M)+\sum_{i=0}^{M-1} d(i, M)
$$

Suppose a new vertex $M$ attaches to an $s^{-}$complete graph and this complete graph comprises vertices of $w_{1}, w_{2}, \cdots w_{m}$, we can therefore rewrite (15) as:

$$
\sigma(M+1)=\sigma(M)+M+\sum_{i=1}^{M-1} D(i, w)
$$

Where, $D(i, w)=\min \left(d\left(i, w_{1}\right), d\left(i, w_{2}\right), L, d\left(i, w_{s}\right)\right)$.

Suppose $\psi$ is the number of vertex set $\left\{w_{1}, w_{2}, \cdots, w_{s}\right\}$ in vertex set $\{0,1, \cdots, M-1\}$, and we see $\sum_{i \in \psi} d(i, w)$ as the total distance of a given vertex $w$ to other vertices in an $N-s+1$ vertex network. Thus $\sum_{i \in \psi} d(i, w)$ can be approximately expressed as:

$$
\sum_{i \in \psi} d(i, w) \approx(M-s) L(M-s+1)
$$

And we $D(i, w)$ is close to $d(i, w)$, so we have:

$$
\sigma(M+1)=\sigma(M)+M+\sum_{i \in \psi} d(i, w)
$$

Apparently, $L(M)$ is a monotonically increased function. When $s \geq 2$, we can have:

$$
(M-s) L(M-s+1)=\frac{2 \sigma(M-s+1)}{M-s+1}<\frac{2 \sigma(M)}{M}
$$

Noticing $\sigma(M+1) \approx \sigma(M)+M+\sum_{i \in \psi} d(i, w)$, an inequality (20) can be obtained:

$$
\sigma(M+1)<\sigma(M)+M+\frac{2 \sigma(M)}{M}
$$

We alter (20) into a differential equation:

$$
\frac{d \sigma(M)}{d M}<M+\frac{2 \sigma(M)}{M}
$$

When $\frac{d \sigma(M)}{d M}=M+\frac{2 \sigma(M)}{M}$, the solution to equation is:

$$
\sigma(M)=M^{2} \ln M+\eta
$$

Where, $\eta$ is a constant. We can get the average path length:

$$
L(M)=\frac{2 \sigma(M)}{M(M-1)}<\frac{2\left(M^{2} \ln (M)+\mu\right)}{M(M-1)}
$$

When $N \rightarrow \infty, L(M)$ will at most increase with the rate of $\ln (N)$, i.e., $L(M) \square \ln (M)$.

We then proceed the simulation of $L(M)$ according to (19), and we set initial $s=3$ and $s=4$. The simulations illustrated as fig. 5. From fig. 5, we know that the average path length follows power-law distribution, and the average path length increases very slowly no matter $s=3$ or $s=4$, implying that one member can averagely find others in a very short distance. Short average path length is another property of small network. 


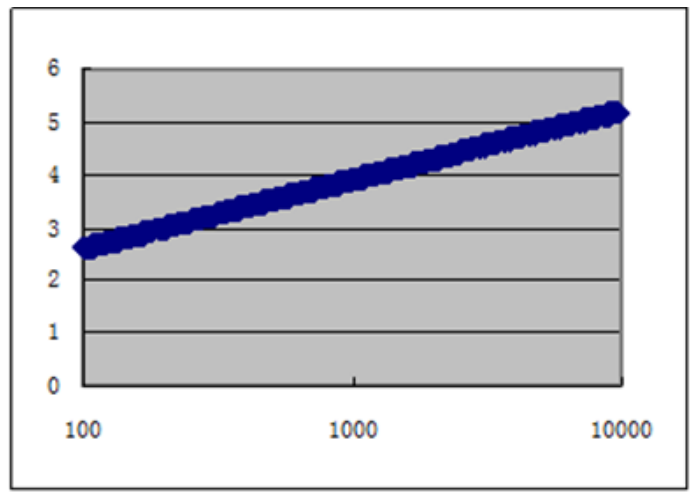

(a) $s=3$

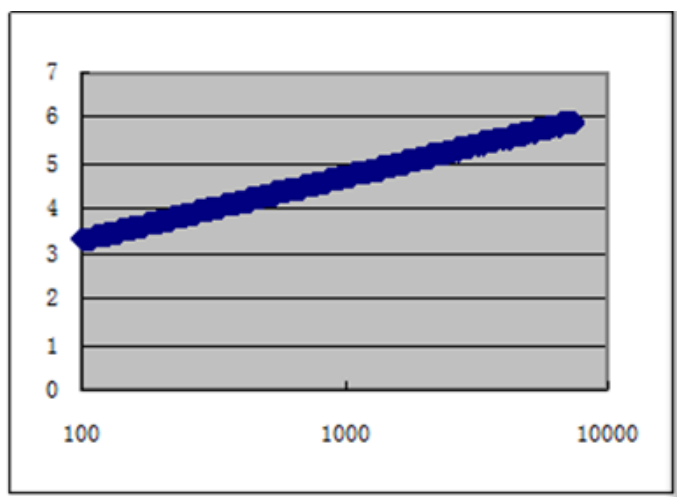

(b) $s=4$

Figure 5. The average path length in the evolving network

Short average path length is very essential for self-employment activities. Statistics show that most members will only seek for help from their relatives or acquaintance, so length lager than 3 is not feasible for entrepreneurship (Yang X., 2012). Fortunately, in our research, most of members can find their targets in less than 3 steps. Agglomeration in this paper is efficient in fostering new entrepreneurial behaviors. However, short length will probably cut down the exclusion of knowledge transferring, so it is not efficient in protecting business secrets and business property rights. Thus, most of the entrepreneurship in self-employment agglomeration is low-tech based and homogeneity based. They are fragile in resisting market risks and less capable in fostering sustainable advantages.

\section{Conclusion}

Self-employment agglomeration is a very typical entrepreneurship in China's urbanization process. Without tedious and strict permission conditions, new comers can basically obtain resource aids even when they are facing insufficient resource endowment because the communications in agglomeration is very frequent. From 2 topologies of investigated self-employment agglomerations in China, and referring to complex network theory, we construct an evolving network which can basically reflect the situation of agglomeration evolution in China. After giving preferential attachment rule, we firstly simulate its evolving process; and then, from the dynamic perspective, we calculate this evolving network's statistic properties and discuss the mechanism of statistic properties on self-employment.

As for average degree, self-employment agglomeration with this evolving rule has comparatively big average degree, which is beneficial for resource sharing in entrepreneurship. As for degree distribution, an agglomeration with given attachment rules has the power-law degree distribution, so it belongs to scale-free network. As for clustering coefficient, whole network with preferential attachment rules in this paper has very large clustering coefficient. As for average path length, it follows power-law distribution, and the average path length is short. Large clustering coefficient and short average path length indicates that this agglomeration has small world properties.

For its free scale and small world properties, the self-employment agglomeration is beneficial for resource sharing, and its members can easily get aid from inner and intra entities. These properties are very essential for necessity entrepreneurs because they are eager for resource compensation from outside. Thus more support from authorities will benefit the self employment performance. However, excessive communication among entities in this kind of agglomeration can not stimulate innovation and guarantee property rights for entrepreneurs, which may affect the efficiency and sustainability in business development. For authorities, they should tighten property law enforcement when they introduce more innovation roles to the agglomerations.

\section{Acknowledgements}

This paper was supported by National Social Science Foundation of China (Grant No. 13CGL022), Research Foundation of Education Bureau of Hunan Province, China (Grant No. 15B046), also financed by Hunan New Urbanization Research Base.

\section{References}

A. Katz, J., \& M. Williams, P. (1997). Gender, self-employment and weak-tie networking through formal organizations. Entrepreneurship \& Regional Development, 9(3), 183-198. http://dx.doi.org/10.1080/08985629700000010

Albert R, Barabási A L (2002). Statistical mechanics of complex networks. Reviews of Modern Physics, 74(1), 47-97. 
http://dx.doi.org/10.1103/RevModPhys.74.47

Aldrich H E, Kim P H (2007). Small worlds, infinite possibilities? How social networks affect entrepreneurial team formation and search. Strategic Entrepreneurship Journal, 1(1-2),147-165. http://dx.doi.org/10.1002/sej.8

Alvarez S A, Busenitz L W (2001). The entrepreneurship of resource-based theory. Journal of Management, 27(6),755-775. http://dx.doi.org/10.1007/978-3-540-48543-8_10

Audretsch, D., \& Keilbach, M. (2004). Entrepreneurship capital and economic performance. Regional studies, 38(8), 949-959. http://dx.doi.org/10.1080/0034340042000280956

Barabási A L, Albert R, Jeong H (1999). Mean-field theory for scale-free random networks]. Physica A: Statistical Mechanics and its Applications, 272(1),173-187. http://dx.doi.org/10.1016/S0378-4371(99)00291-5

Davidsson, P., \& Honig, B. (2003). The role of social and human capital among nascent entrepreneurs. Journal of business venturing, 18(3), 301-331. http://dx.doi.org/10.1016/S0883-9026(02)00097-6

Dobusch, L., \& Schüßler, E. (2013). Theorizing path dependence: a review of positive feedback mechanisms in technology markets, regional clusters, and organizations. Industrial and Corporate Change, 22(3), 617-647. http://dx.doi.org/10.1093/icc/dts029

Dorogovtsev S N, Mendes J F F, Samukhin A N (2000). Structure of growing networks with preferential linking. Physical Review Letters, 85(21), 4633-4636. http://dx.doi.org/10.1103/PhysRevLett.85.4633

Ferriani, S., Cattani, G., \& Baden-Fuller, C. (2009). The relational antecedents of project-entrepreneurship: Network centrality, team composition and project performance. Research Policy, 38(10), 1545-1558. http://dx.doi.org/10.1016/j.respol.2009.09.001

Hernandez L, Nunn N, Warnecke T (2012). Female entrepreneurship in China: opportunity-or necessity-based?. International Journal of Entrepreneurship and Small Business, 15(4),411-434. http://dx.doi.org/10.1504/IJESB.2012.046473

Hoang, H., \& Yi, A. (2015). Network-based Research in Entrepreneurship: A Decade in Review. Foundations and Trends (R) in Entrepreneurship, 11(1), 1-54. http://dx.doi.org/10.1561/0300000052

Jack S L, Anderson A R (2002). The effects of embeddedness on the entrepreneurial process. Journal of Business Venturing, 17(5): 467-487. http://dx.doi.org/10.1016/S0883-9026(01)00076-3

Kim P. H., Aldrich H. E., Keister L. A. (2006). Access (not) Denied: the Impact of Financial, Human, and Cultural Capital on Entrepreneurial Entry in the United States. Small Business Economics, 27, 5-22. http://dx.doi.org/10.1007/s11187-006-0007-x

Kloosterman, R. C. (2010). Matching opportunities with resources: A framework for analysing (migrant) entrepreneurship from a mixed embeddedness perspective. Entrepreneurship and Regional Development, 22(1), 25-45. http://dx.doi.org/10.1080/08985620903220488

Puga D., Venables A.J.(1996). The Spread of Industry: Spatial Agglomeration in Economic Development[J]. Journal of the Japanese and International Economies, (10), 440-464. http://dx.doi.org/10.1006/jjie.1996.0025

Rosenthal S. S., Strange W. C. (2003). Geography, Industrial Organization, and Agglomeration[J]. Review of Economics and Statistics, 85, 377-393. http://dx.doi.org/10.1162/003465303765299882

Savvas P., Feng Li, Henry Etzkowitz et al. (2009). Entrepreneurial Networks: A Triple Helix Approach for Brokering Human and Social Capital. Journal of International Entrepreneurship, 7(3): 215-235. http://dx.doi.org/10.1007/s10843-009-0038-x

Tang Y (2013). The Structure Comparison of Necessity Entrepreneurial Resource Support Networks Based on SNA. Business and Management Research, 2(3),137-145. http://dx.doi.org/10.5430/bmr.v2n3p137

Wiklund, J., Davidsson, P., Audretsch, D. B., \& Karlsson, C. (2011). The future of entrepreneurship research. Entrepreneurship Theory and Practice,35(1), 1-9. http://dx.doi.org/10.1111/j.1540-6520.2010.00420.x

Yang X, Ho E Y H, Chang A (2012). Integrating the resource-based view and transaction cost economics in immigrant business performance. Asia Pacific Journal of Management, 29(3),753-772. http://dx.doi.org/10.1007/s10490-010-9236-2

Yong T (2011). Exploration on Social Capital Dimensions in Necessity Entrepreneurship: Based on the Data from Migrant Workers' View. Journal of Hunan City University, 32(5), 49-53 . (in Chinese)

Zhang Z Z, Rong L L, Zhou T (2005). An evolving model for scale-free collaboration networks. System Engineering Theory and Practice, (11), 55-60. (in Chinese) 